BNL-114337-2017-JA

\title{
An Ideal Electrode Material, 3D Surface-microporous Graphene for Supercapacitors with Ultrahigh Areal Capacitance
}

\author{
Dario J. Stacchiola \\ Submitted to the Journal of ACS Applied Materials \& Interfaces
}

July 2017

Center for Functional Nanomaterials

Brookhaven National Laboratory

U.S. Department of Energy
USDOE Office of Science (SC),
Basic Energy Sciences (BES) (SC-22)

Notice: This manuscript has been authored by employees of Brookhaven Science Associates, LLC under Contract No. DE- SC0012704 with the U.S. Department of Energy. The publisher by accepting the manuscript for publication acknowledges that the United States Government retains a non-exclusive, paid-up, irrevocable, world-wide license to publish or reproduce the published form of this manuscript, or allow others to do so, for United States Government purposes. 


\section{DISCLAIMER}

This report was prepared as an account of work sponsored by an agency of the United States Government. Neither the United States Government nor any agency thereof, nor any of their employees, nor any of their contractors, subcontractors, or their employees, makes any warranty, express or implied, or assumes any legal liability or responsibility for the accuracy, completeness, or any third party's use or the results of such use of any information, apparatus, product, or process disclosed, or represents that its use would not infringe privately owned rights. Reference herein to any specific commercial product, process, or service by trade name, trademark, manufacturer, or otherwise, does not necessarily constitute or imply its endorsement, recommendation, or favoring by the United States Government or any agency thereof or its contractors or subcontractors. The views and opinions of authors expressed herein do not necessarily state or reflect those of the United States Government or any agency thereof. 


\title{
An Ideal Electrode Material, 3D Surface-microporous Graphene for Supercapacitors with Ultrahigh Areal Capacitance
}

Liang Chang, Dario J. Stacchiola and Yun Hang Hu*

\author{
L. Chang, Prof. Y. H. Hu \\ Department of Materials Science and Engineering, Michigan Technological University, 1400 \\ Townsend Drive, Houghton, MI 49931-1295, USA \\ E-mail: yunhangh@mtu.edu \\ Dr. D. J. Stacchiola \\ Center for Functional Nanomaterials, Brookhaven National Laboratory, Upton, NY 11973, \\ USA
}

Keywords: cabbage-coral-like graphene, surface micropores, electric double-layer capacitors, ultrahigh areal capacitance

The efficient charge accumulation of an ideal supercapacitor electrode requires abundant micropores and its fast electrolyte-ions transport prefers meso/macro pores. However, current electrode materials cannot meet both requirements, resulting in poor performance. Herein, we creatively constructed three-dimensional cabbage-coral-like graphene as an ideal electrode material, in which meso/macro channels are formed by graphene walls and rich micropores are incorporated in the surface layer of the graphene walls. The unique 3D graphene material can achieve high gravimetric capacitance of $200 \mathrm{~F} / \mathrm{g}$ with aqueous electrolyte, three times larger than that of commercially used activated carbon $(70.8 \mathrm{~F} / \mathrm{g})$. Furthermore, it can reach an ultrahigh areal capacitance of $1.28 \mathrm{~F} / \mathrm{cm}^{2}$ and excellent rate capability (83.5\% from 0.5 to 10 $\mathrm{A} / \mathrm{g}$ ) as well as high cycling stability (91.3\% retention after 3000 cycles). The excellent electric double-layer performance of the 3D graphene electrode can be attributed to the fast electrolyte ion-transport in the meso/macro channels and the rapid and reversible charge adsorption with negligible transport distance in the surface-micropores.

Electric double-layer capacitors (EDLCs) store energy through electrostatic charge accumulations on the surface of porous electrodes under applied voltage. ${ }^{[1]}$ EDLCs possess 
higher energy density than conventional capacitors and larger power density than batteries with excellent reversibility and long-life expectation as well as wide range operation. ${ }^{[2-5]}$ Those unique features make EDLCs an indispensable part of renewable energy systems to collect and utilize intermittent wind power, hydro power, and solar energy. ${ }^{[6-9]}$.

EDLCs are usually composed of two symmetrically porous carbon electrodes with a separator and electrolyte between them. The performance of EDLCs is strongly dependent on the surface area and pore structure of carbon electrodes. ${ }^{[10-12]}$ It is generally recognized that micropores are beneficial for charge accumulation to form electric double-layers due to enhanced surface area and enriched active sites. ${ }^{[13,14]}$ However, microporous electrode materials often suffer the inhibited transport of electrolyte ions, leading to inaccessible surface areas and thus poor electrochemical behavior. Activated carbon (AC), which is representative microporous material with surface areas up to thousands $\mathrm{m}^{2} / \mathrm{g}$, is widely used in commercialized EDLCs. However, the capacitance of AC electrodes possesses a limited capacitance of 40-70 F/g. Great efforts were made to improve its performance by expanding the micropores to meso/macropores, but the enhancement is far below expectation. ${ }^{[15-18]}$ To achieve better EDLC performance, an ideal electrode material should possess a large proportion of micropores for efficient electric double-layer formation without the transport limitation issue of electrolyte ions in micropores. ${ }^{[19]}$

Graphene has attracted much attention for supercapacitors due to its unique mechanical and electrical properties as well as large surface area. A single layer graphene sheet possesses a large theoretical surface area of $2630 \mathrm{~m}^{2} / \mathrm{g}$ with an ultrahigh electrical conductivity, predicting a high theoretical capacitance of $526 \mathrm{~F} / \mathrm{g} .{ }^{[20]}$ However, so far, the obtained capacitance of 2Dgraphene-based supercapacitors is still far below the theoretical value (especially for a large mass loading), because the aggregation of graphene sheets inhibits electrolyte ions to contact all surface area. ${ }^{[21,22]}$ Several strategies were exploited to solve the issues. Ruoff et al. pioneered chemically modified graphene (CMG) for supercapacitor electrodes, leading to the gravimetric 
capacitance of $135 \mathrm{~F} / \mathrm{g}$ at current of $10 \mathrm{~mA}$ in $5.5 \mathrm{M} \mathrm{KOH} .{ }^{[23]}$ Furthermore, they obtained a better capacitance of $165 \mathrm{~F} / \mathrm{g}$ at $1.4 \mathrm{~A} / \mathrm{g}$ in $\left(\mathrm{BMIM} \mathrm{BF}_{4}\right) / \mathrm{AN}$ electrolyte by activating graphene with $\mathrm{KOH}$ oxidant. ${ }^{[24]}$ Shi et al constructed a self-assembly graphene hydrogel (SGH) for supercapacitors, exhibiting the specific capacitance of $175 \mathrm{~F} / \mathrm{g}$ at scan rate of $10 \mathrm{mV} / \mathrm{s}$ in $5 \mathrm{M}$ $\mathrm{KOH} .{ }^{[25]}$ Kaner et al. demonstrated an areal capacitance of $1.84 \mathrm{mF} / \mathrm{cm}^{2}$ at a ultrahigh rate of $1000 \mathrm{~A} / \mathrm{g}$ with laser reduced graphite oxide electrodes in a symmetrically flexible supercapacitor. ${ }^{[26]} \mathrm{Li}$ et al. synthesized an electrolyte-mediated chemically converted graphene (EM-CCG) to create a continuous ion transport network in dense packing electrodes, leading to exceptional high volumetric capacitance and volumetric energy density in $\mathrm{EMIMBF}_{4} /$ acetonitrile (AN). ${ }^{[27]}$ Recently, we synthesized a new type of graphene sheets with $3 \mathrm{D}$ honeycomb-like structure (HSG) via an invented reaction between $\mathrm{Li}_{2} \mathrm{O}$ and $\mathrm{CO}{ }^{[28]}$ Furthermore, we combined our strengths in the synthesis of 3D graphene sheets and the conversion of $\mathrm{CO}_{2}$ greenhouse gas, leading to the successful synthesis of 3D cauliflowerfungus-like graphene (CFG) directly from $\mathrm{CO}_{2}$ via one step reaction. ${ }^{[29]}$ The 3D CFG was explored for supercapacitors, resulting in gravimetric capacitance of $112.4 \mathrm{~F} / \mathrm{g}$ (with total surface area of $462 \mathrm{~m}^{2} / \mathrm{g}$ ). Furthermore, we synthesized porous Na-embedded carbon nanowalls (Na@C) and employed it for supercapacitors, leading to gravimetric capacitance of $143.2 \mathrm{~F} / \mathrm{g}$ (with total surface area of $555 \mathrm{~m}^{2} / \mathrm{g}$ ). ${ }^{[30]}$ Although those two carbon nanomaterials are mesoporous materials with fully accessible surface areas, their total surface areas are not high enough to obtain very high capacitance. This has stimulated us to design and synthesize new types of graphene materials for better electrochemical performance. Herein, we demonstrate a new strategy to create a novel 3D surface-microporous graphene (SMG), which can meet two requirements for an ideal supercapacitor electrode: its fully accessible surface-micropores ensure efficient charge accumulation and its meso/macro channels provide fast paths for electrolyte-ion transport. As a result, a high gravimetric capacitance of 200 F/g was obtained 
with $83.5 \%$ retention associated with 20 times increase in current density. Furthermore, the SMG electrode exhibited an ultrahigh areal capacitance of $1.28 \mathrm{~F} / \mathrm{cm}^{2}$.

We invented the reaction between $\mathrm{Na}$ liquid and $\mathrm{CO}_{2}$ gas for graphene synthesis:[31]

$$
4 \mathrm{Na}(\mathrm{l})+3 \mathrm{CO}_{2}(\mathrm{~g})=\mathrm{C}(\text { graphene })(\mathrm{s})+2 \mathrm{Na}_{2} \mathrm{CO}_{3}(\mathrm{~s})
$$

The reaction is thermodynamically favorable with negative Gibbs free energy change (-626.721 $\mathrm{kJ} / \mathrm{mol})$ and enthalpy change $(-1063.256 \mathrm{~kJ} / \mathrm{mol})$. Herein, based on this reaction, we propose a simple and novel approach to synthesize a novel material, 3D surface-microporous graphene (SMG): (1) $\mathrm{CO}_{2}$ reacts with $\mathrm{Na}$ liquid to generate graphene sheets and $\mathrm{Na}_{2} \mathrm{CO}_{3}$ nano particles, (2) the produced $\mathrm{Na}_{2} \mathrm{CO}_{3}$ plays a role to prevent the aggregation of graphene sheets and determine their shapes, (3) $\mathrm{CO}_{2}$ can in-situ oxidize graphene walls to create surface micropores, and (4) the obtained solid products can be treated by aqueous solution of $\mathrm{HCl}$ to remove $\mathrm{Na}_{2} \mathrm{CO}_{3}$ nanoparticles, generating meso/macro channels in the 3D graphene. The feasibility of this strategy was confirmed by the following experiments.

Na cubes were loaded into a ceramic tube reactor and 50 psi $\mathrm{CO}_{2}$ was then introduced into the reactor, followed by increasing temperature to $520^{\circ} \mathrm{C}$ and maintaining the temperature for $12 \mathrm{~h}$. The solid products were treated with $37 \mathrm{wt} \% \mathrm{HCl}$ overnight, followed by deionized water washing for several times until $\mathrm{PH}=7$ and drying at $80^{\circ} \mathrm{C}$ for overnight. The obtained black carbon product was subjected to characterizations. Field emission scanning electron microscopy (FESEM) image shows cabbage-coral-like graphene with meso/macro channels (Figure 1A). The widths of the channels (which were built by coiled and bended graphene sheets) are ranged from $200 \mathrm{~nm}$ to $1 \mathrm{um}$. The 3D cabbage-coral-like structure was further confirmed by transmission electron microscopy (TEM) image (Figure 1B). Each of graphene sheets is $2.2 \mathrm{~nm}$ thick. Furthermore, the lattice spacing of graphene layers, which was calculated from XRD pattern (Figure 1C), is $3.55 \AA$. Therefore, each of graphene sheet consists of 6 graphene layers. 
The surface area and pore structure of the 3D graphene were evaluated by $\mathrm{N}_{2}$ adsorption at $77 \mathrm{~K}$ (Figure 1D). Its total surface area is $890 \mathrm{~m}^{2} / \mathrm{g}$, in which micropores contribute $789 \mathrm{~m}^{2} / \mathrm{g}$ as microporous surface area. This indicates that almost $90 \%$ surface area of the graphene originates from micropores. The average diameter of micropores, which was obtained from $\mathrm{N}_{2}$ adsorption measurement, is $1.6 \mathrm{~nm}$. To estimate the average deepness of micropores, we treat the micropores as typical cylinder pores and thus express their wall surface area $\left(S_{\text {wall }}\right)$ (excluding their bottom area) as

$$
S_{\text {wall }}=n \pi D h
$$

where $n$ is the number of micropores per gram graphene, $D$ the average diameter of micropores, and $h$ the average deepness of the micropores. The number of micropores per gram graphene and the wall surface area are $1.68 \times 10^{20}(1 / \mathrm{g})$ and $452\left(\mathrm{~m}^{2} / \mathrm{g}\right)$, respectively (see suplymentary information). Using Equation 1, we obtained a very small average deepness (h) of $0.54 \mathrm{~nm}$, indicating that the micropores are only in the surface graphene layer. We can illuminate this novel 3D SMG material in Figure 2. The bended and coiled graphene sheets constitute interconnected meso/macro channels with cabbage-coral-like shape, which can buffer electrolyte as a reservoir due to fast transport of electrolyte ions. Furthermore, the buffered electrolyte ions can rapidly diffuse to the surface-micropores of the graphene walls with negligible transport distance in the pores, forming efficient electric double-layers. Thus, the 3D SMG electrode can be expected to achieve high capacitance and excellent rate performance for EDLCs.

To experimentally demonstrate its excellent electrochemical performance, 3D SMG was used to fabricate two identical electrodes for a symmetrically two-electrode supercapacitor cell with a glassy fiber separator and $2 \mathrm{M} \mathrm{KOH}$ electrolyte. As shown in Figure 3A, one can see that cyclic voltammetry (CV) curve of 3D SMG electrodes possesses ideal rectangular shape, indicating reversible charge adsorption/desorption process. The excellent electric double-layer 
behavior is further confirmed by the symmetrically triangle in the galvanostatic charge/discharge profile (Figure 3B). As a surface-microporous material, 3D SMG (200 F/g) reached three times larger gravimetric capacitance than conventional-microporous AC (70.8 F/g) at current density of $0.5 \mathrm{~A} / \mathrm{g}$, although both materials have comparable surface areas. The excellent EDLC performance of 3D SMG can be maintained even when the current density increases to $2 \mathrm{~A} / \mathrm{g}$ (Figure 3C). The huge difference in EDLC performance between 3D SMG and AC electrodes is also reflected by CV areas (Figure 3A) and charge/discharge times (Figure 3B). Furthermore, Nyquist plots were employed to evaluate ion diffusions in the SMG electrodes, because the greater slope in low frequency region would reflect a faster ion diffusion. ${ }^{[32]}$ As shown in Figure 3D, one can see the slope in low frequency region is much larger for the SMG electrode than for the AC electrode, indicating that the SMG electrode possess a faster ion-diffusion rate than the AC electrode. If total surface areas of AC $\left(853 \mathrm{~m}^{2} / \mathrm{g}\right)$ and 3D SMG $\left(890 \mathrm{~m}^{2} / \mathrm{g}\right)$ are taken into account, theoretical specific gravimetric capacitances of AC and 3D SMG electrodes are 170.1 and $178.0 \mathrm{~F} / \mathrm{g}$, respectively. ${ }^{[29]}$ Theoretical capacitance of the AC electrode is much larger than its experimental value $(70.8 \mathrm{~F} / \mathrm{g})$, indicating that most of AC surface area is not accessible for electrolyte ions. In contrast, all surface area of SMG is fully accessible for electrolyte ions, reflected by larger specific gravimetric capacitance (200 F/g) of 3D SMG than its theoretical value. Those indicate that, totally different from conventional micropores, SMG surface-micropores can be fully utilized for charge accumulation to form efficient electric double-layers. This happened because the transport distance of electrolyte ions is negligible in the surface-micropores due to their very small deepness $(0.54 \mathrm{~nm})$. The excellent electric double-layer behavior of the 3D SMG electrodes was further demonstrated by following observations: only slight polarization in CV curves and invisible IR drop appeared in galvanostatic charge/discharge profiles, even when scan rate was increased from 30 to $300 \mathrm{mV} / \mathrm{s}$ or current density from $0.5 \mathrm{~A} / \mathrm{g}$ to $10 \mathrm{~A} / \mathrm{g}$ (Figure 4A and 4B). Furthermore, the 3D SMG electrode exhibited excellent rate capability, namely, when current 
density was increased by 20 times, the capacitance decreased from $200 \mathrm{~F} / \mathrm{g}$ to $167 \mathrm{~F} / \mathrm{g}$, indicating 83.5\% capacitance retention (Figure 4C). In addition, the 3D SMG electrode is very stable. After 3000 galvanostatic charge/discharge cycles at current density of $10 \mathrm{~A} / \mathrm{g}$, capacitance retention is as large as $91.3 \%$ (Figure 4D).

The 3D SMG electrode also showed excellent scalability for supercapacitors. When the mass loading of the 3D SMG electrode increased from T1 $\left(2.8 \mathrm{mg} / \mathrm{cm}^{2}\right)$ to T2 $\left(6.4 \mathrm{mg} / \mathrm{cm}^{2}\right)$, the rectangular $\mathrm{CV}$ curve at $20 \mathrm{mV} / \mathrm{s}$ and symmetrical charge/discharge triangle curve at $2 \mathrm{~A} / \mathrm{g}$ remained unchanged (Figure 5A and 5B), leading to almost constant capacitance (namely, from $200 \mathrm{~F} / \mathrm{g}$ to $199.7 \mathrm{~F} / \mathrm{g}$ ). When the current density was increased by 10 times, the electrode still showed excellent electrochemical performance (Figure 5C). This indicates that the 3D SMG electrode is suitable for fast charging/discharging even with high mass loading. Furthermore, the scalable feature of the SMG electrode creates its ultrahigh areal capacitance of $1.28 \mathrm{~F} / \mathrm{cm}^{2}$ (Figure 5D).

As shown above, the mass capacitance (200 F/g) of the 3D SMG electrode is even larger than its theoretic value (178.0 F/g). The exceeding capacitance over the theoretical value might be attributed to a redox reaction of functional groups. This was supported by energy dispersive spectroscopy (EDS) measurement, which shows the oxygen content of $4.88 \%$ in the SMG. Furthermore, the oxygen functional groups were further evaluated with X-ray photoelectron spectroscopy (XPS) and Fourier transform infrared spectroscopy (FTIR). As shown in Figure S2A, C1s XPS curve can be deconvoluated into 4 peaks. The two peaks at $286.2 \mathrm{eV}$ and 288.1 $\mathrm{eV}$ can be assigned to $\mathrm{C}-\mathrm{O}$ and $\mathrm{C}=\mathrm{O}$ groups, respectively. The other peaks at $284.2 \mathrm{eV}$ and $290.1 \mathrm{eV}$ are associated with $\mathrm{C}=\mathrm{C}$ and $\pi-\pi^{*}$, respectively. ${ }^{[33]}$ The results can be further verified by Fourier transform infrared spectroscopy (FTIR), namely, the $\mathrm{C}-\mathrm{O}$ and $\mathrm{C}=\mathrm{C}$ groups were further confirmed by their stretching IR bands at $1064 \mathrm{~cm}^{-1}$ and $1537 \mathrm{~cm}^{-1}$ (Figure S2B). ${ }^{[34]}$ Those oxygen functional groups in the 3D SMG not only provide redox capacitance, but also improve wettability and thus benefit electrochemical performance. 
In summary, a novel strategy was demonstrated to synthesize an ideal supercapacitor electrode material, 3D surface-microporous graphene (3D SMG) with cabbage-coral-like structure. In this unique material, the meso/macro channels (formed by graphene walls) provide excellent paths for fast electrolyte ion transport and the rich micropores in the graphene surface layer are efficient for charge accumulation to form efficient electric double-layers. Consequently, the 3D SMG electrode can achieve high gravimetric capacitance of $200 \mathrm{~F} / \mathrm{g}$ with aqueous electrolyte, three times larger than that of commercially used activated carbon (70.8 F/g). Furthermore, it exhibited an ultrahigh areal capacitance of $1.28 \mathrm{~F} / \mathrm{cm}^{2}$ and excellent rate capability (83.5\% from 0.5 to $10 \mathrm{~A} / \mathrm{g}$ ) as well as impressive cycling stability (91.3\% retention after 3000 cycles).

\section{Supporting Information}

Supporting Information is available from the Wiley Online Library or from the author.

\section{Acknowledgements}

This work was supported by U.S. National Science Foundation (CBET-0931587). L.C and Y.H.H. also thank Charles and Carroll McArthur for their great support.

Received: ((will be filled in by the editorial staff))

Revised: ((will be filled in by the editorial staff)) Published online: ((will be filled in by the editorial staff))

\section{References}

[1] E. Lim, C. Jo, J. Lee, Nanoscale 2016, 8, 7827.

[2] L. Mai, X. Tian, X. Xu, L. Chang, L. Xu, Chem. Rev. 2014, 114, 11828.

[3] Q. Wang, J. Yan, Z. Fan, Energy Environ. Sci. 2016, 9, 729.

[4] L. Chang, L. Mai, X. Xu, Q. An, Y. Zhao, D. Wang, X. Feng, RSC Adv. 2013, 3, 1947.

[5] J. H. Won, H. M. Jeong, J. K. Kang, Adv. Energy Mater. 2017, 7, 1601355. 
[6] N. S. Choi, Z. Chen, S. A. Freunberger, X. Ji, Y. K. Sun, K. Amine, G. Yushin, L. F. Nazar, J. Cho, P. G. Bruce, Angew. Chem. Int. Ed. 2012, 51, 9994.

[7] H. Kim, J. Hong, K. Y. Park, H. Kim, S. W. Kim, K. Kang, Chem. Rev. 2014, 114, 11788.

[8] E. Faggioli, P. Rena, V. Danel, X. Andrien, R. Mallant, H. Kahlen, J. Power Sources 1999, 84, 261.

[9] M. Conte, Fuel Cells 2010, 10, 806.

[10] Y. Deng, Y. Xie, K. Zou, X. Ji, J. Mater. Chem. A 2016, 4, 1144.

[11] L. Chang, Y. H. Hu, RSC Adv. 2016, 6, 47285.

[12] G. Wang, L. Zhang, J. Zhang, Chem. Soc. Rev. 2012, 41, 797.

[13] M. Genovese, J. Jiang, K. Lian, N. Holm, J. Mater. Chem. A 2015, 3, 2903.

[14] C. Zhang, W. Lv, Y. Tao, Q. H. Yang, Energy Environ. Sci. 2015, 8, 1390.

[15] S. Yu, Y. Li, N. Pan, RSC Adv. 2014, 4, 48758.

[16] M. Zhang, C. He, E. Liu, S. Zhu, C. Shi, J. Li, Q. Li, N. Zhao, J. Phy. Chem. C 2015, 119, 21810.

[17] E. Y. L. Teo, L. Muniandy, E. Ng, F. Adam, A. R. Mohamed, R. Jose, K. F. Chong, Electrochim. Acta 2016, 192, 110.

[18] Y. Xu, L. Chang, Y. H. Hu, Int. J. Energy Res. 2017, 41, 728.

[19] K. Yuan, Y. Xu, J. Uihlein, G. Brunklaus, L. Shi, R. Heiderhoff, M. Que, M. Forster, T. Chasse, T. Pichler, T. Riedl, Y. Chen, U. Scherf, Adv. Mater. 2015, 27, 6714.

[20] J. Xia, F. Chen, J. Li, N. Tao, Nat. Nanotechnol. 2009, 4, 505.

[21] J. Y. Luo, H. D. Jang, J. X. Huang, ACS Nano 2013, 7, 1464.

[22] H. Jiang, P. S. Lee, C. Z. Li, Energy Environ. Sci. 2013, 6, 41.

[23] M. D. Stoller, S. Park, Y. Zhu, J. An, R. S. Ruoff, Nano Lett. 2008, 8, 3498. 
[24] Y. Zhu, S. Murali, M. D. Stoller, K. J. Ganesh, W. Cai, P. J. Ferreira, A. Pirkle, R. M. Wallace, K. A. Cychosz, M. Thommes, D. Su, E. A. Stach, R. S. Ruoff, Science 2011, 332, 1537.

[25] Y. Xu, K. Sheng, C. Li, G. Shi, ACS Nano 2010, 4, 4324.

[26] M. F. El-Kady, V. Strong, S. Dubin, R. B. Kaner, Science 2012, 335, 1326.

[27] X. Yang, C. Cheng, Y. Wang, L. Qiu, D. Li, Science 2013, 341, 534.

[28] H. Wang, K. Sun, F. Tao, D. J. Stacchiola, Y. H. Hu, Angew. Chem. Int. Ed. 2013, 52, 9210.

[29] L. Chang, W. Wei, K. Sun, Y. H. Hu, J. Mater. Chem. A 2015, 3, 10183.

[30] L. Chang, W. Wei, K. Sun, Y. H. Hu, J. Mater. Chem. A DOI:10.1039/C7TA01085K.

[31] W. Wei. K. Sun, Y. H. Hu, J. Mater. Chem. A 2016, 4, 12054.

[32] B. P. Vinayan, R. Nagar, V. Raman, N. Rajalakshmi, K. S. Dhathathreyan, S. Ramaprahbu, J. Mater. Chem. A 2012, 22, 9949.

[33] A. Sumbaja, C. Y. Foo, X. Wang, P. S. Lee, Adv. Mater. 2013, 25, 2809.

[34] W. Wang, W. Liu, Y. Zeng, Y. Han, M. Yu, X. Lu, Y. Tong, Adv. Mater. 2015, 27, 3572. 

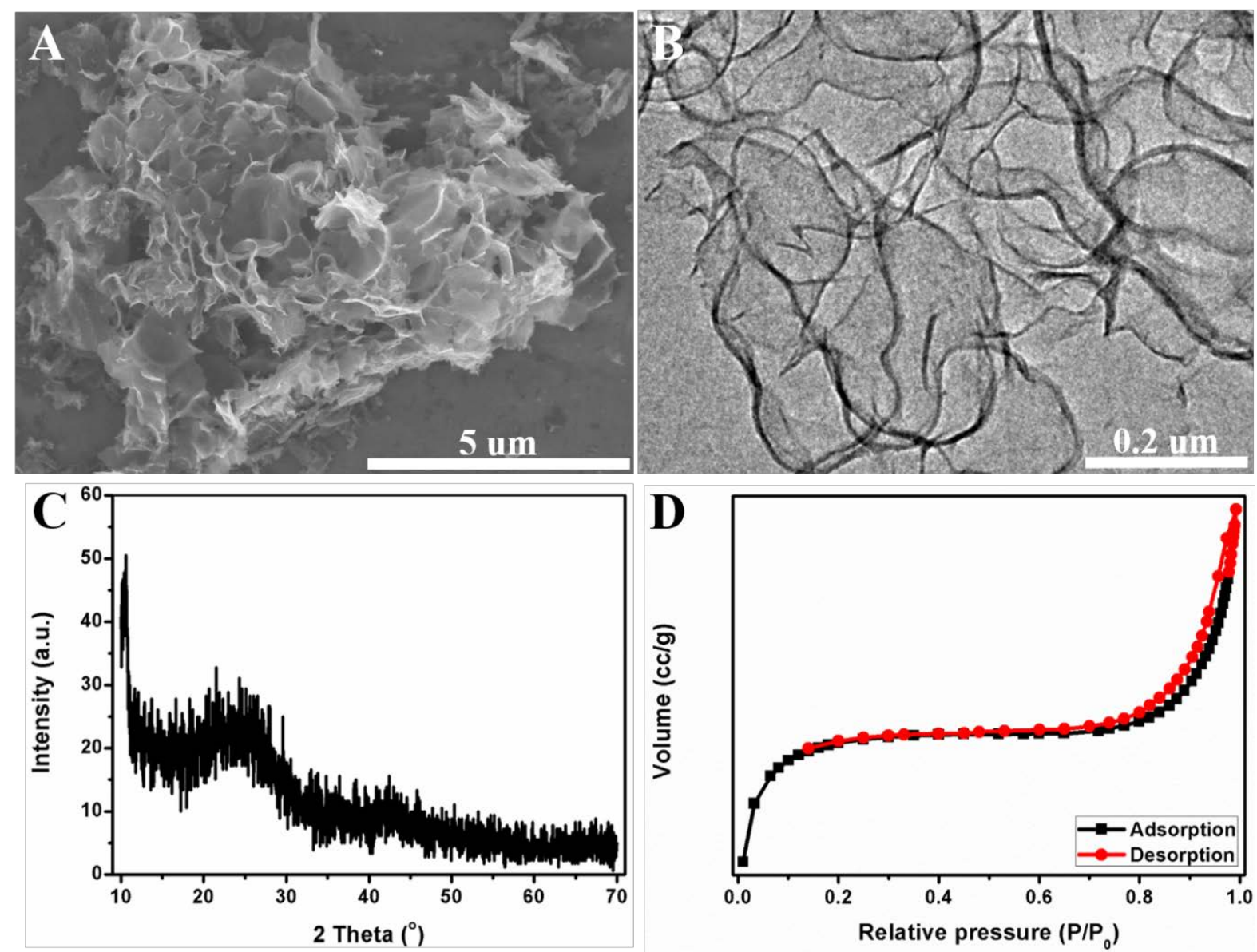

Figure 1. Characterization of 3D SMG: (A) FESEM image, (B) TEM image, (C) XRD pattern, (D) $\mathrm{N}_{2}$ adsorption/desorption curves at $77 \mathrm{~K}$. 


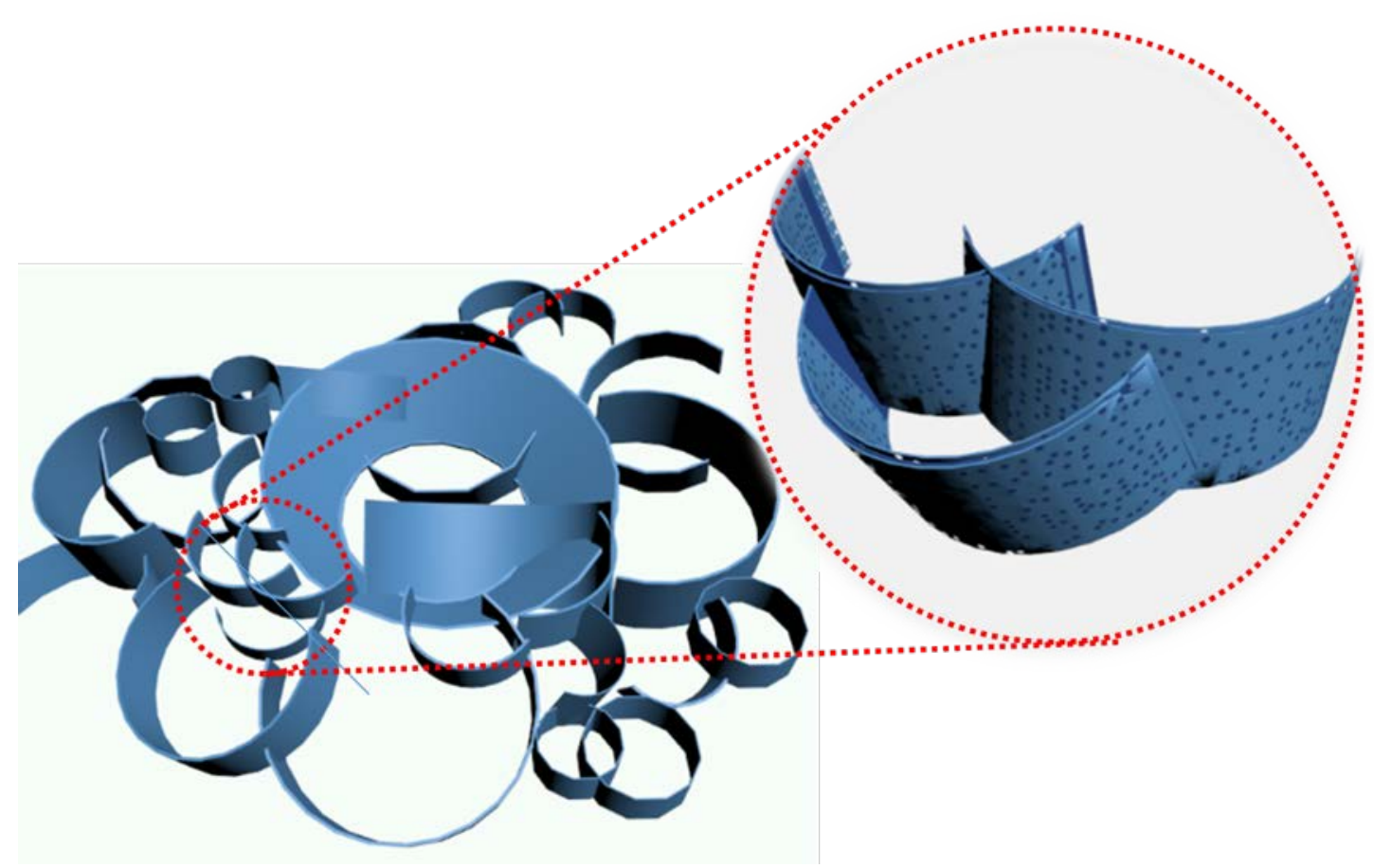

Figure 2. Illustration of 3D SMG structure: Meso/macro channels are formed by graphene walls and micropores are created in the surface layer of the graphene walls. 

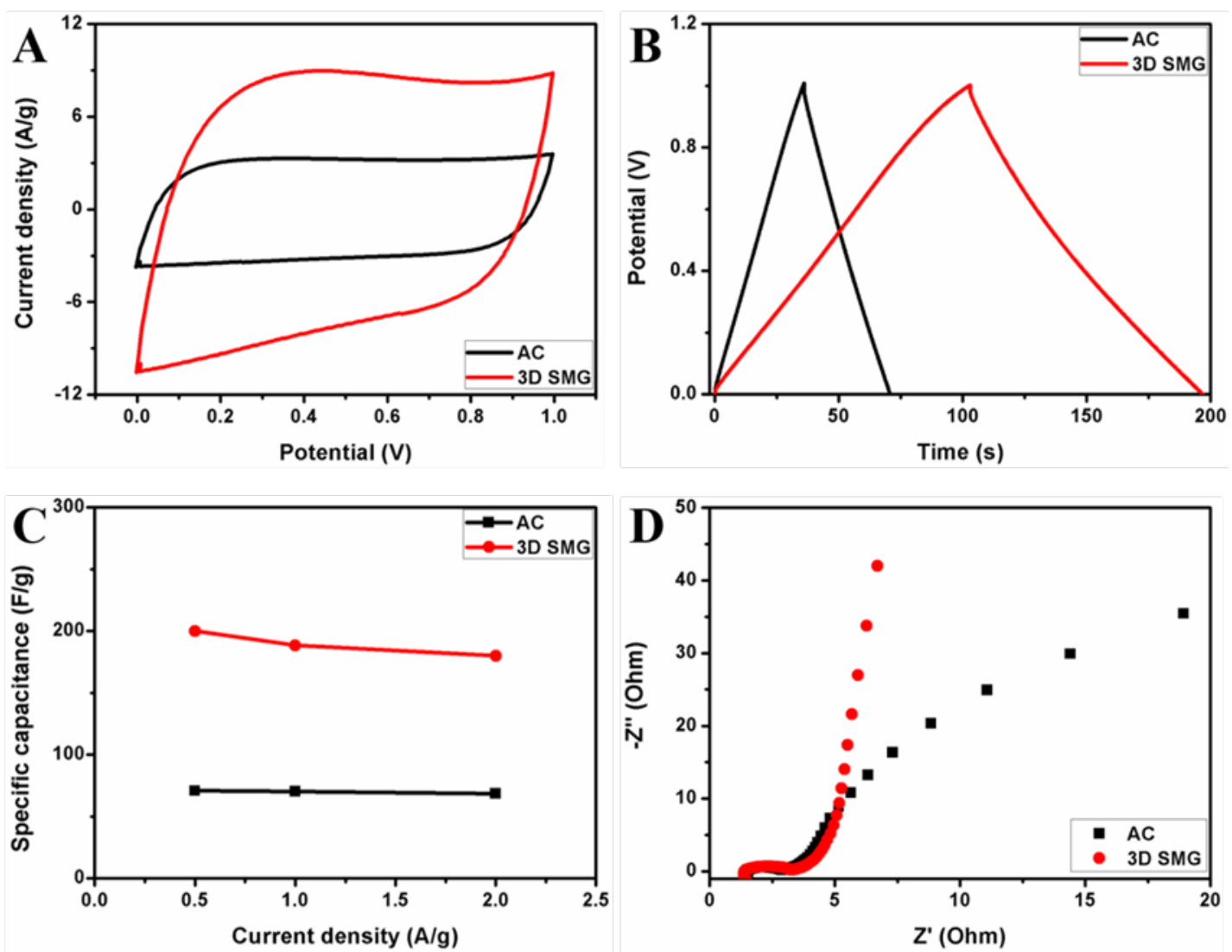

Figure 3. Electrochemical performance of AC and 3D SMG electrodes: (A) CV curves at scan rate of $100 \mathrm{mV} / \mathrm{s}$, (B) galvanostatic charge/discharge profiles at current density of $1 \mathrm{~A} / \mathrm{g},(\mathrm{C})$ current densities vs. specific capacitances, and (D) Nyquist plot with $0.01 \sim 10^{5}$ frequencies. 

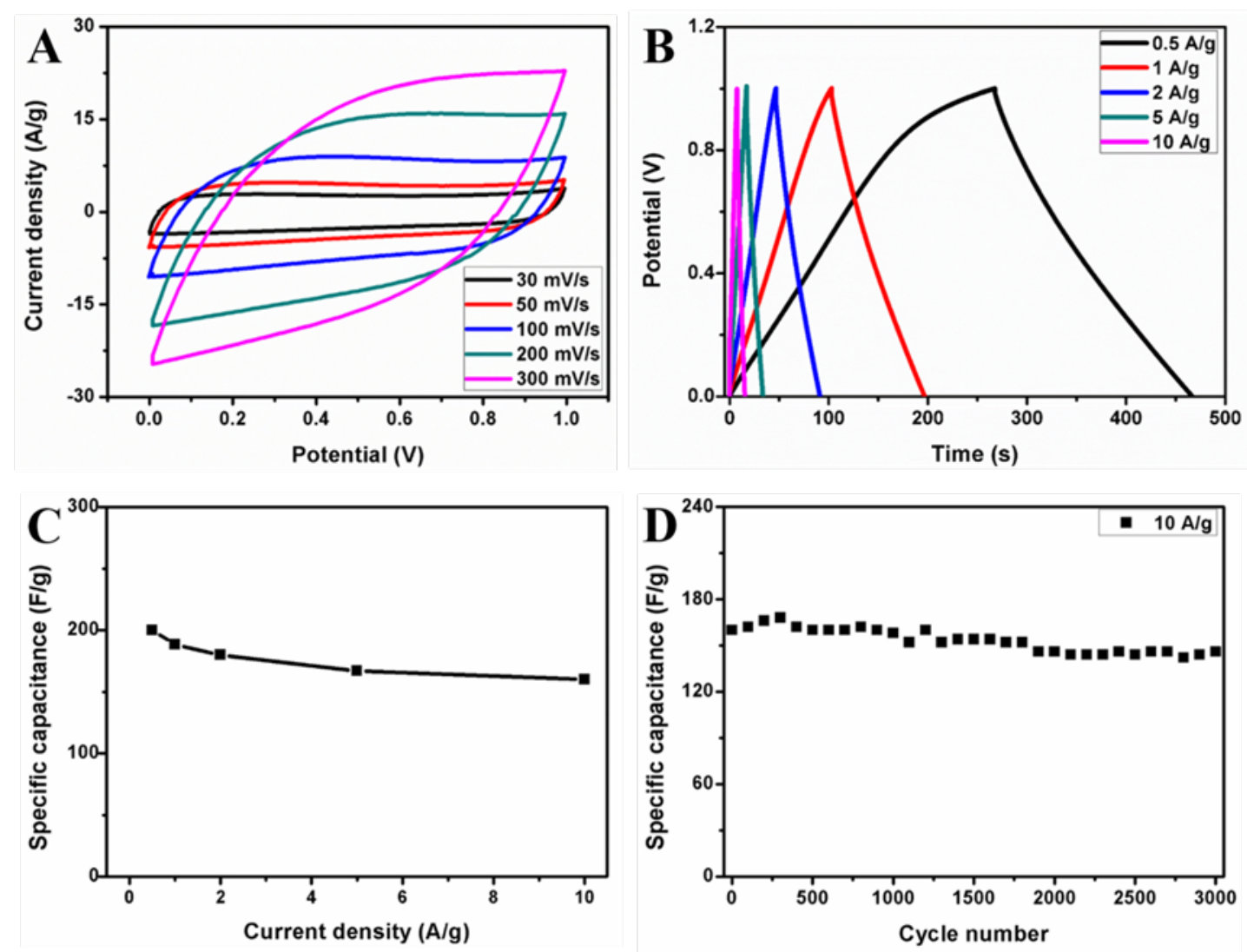

Figure 4. Electrochemical performance of the 3D SMG electrode: (A) CV curves at scan rates from 30 to $300 \mathrm{mV} / \mathrm{s}$, (B) galvanostatic charge/discharge profile at current density of 0.5 10 $\mathrm{A} / \mathrm{g},(\mathrm{C})$ current densities vs. specific capacitances, and (D) cycling performance at $10 \mathrm{~A} / \mathrm{g}$. 

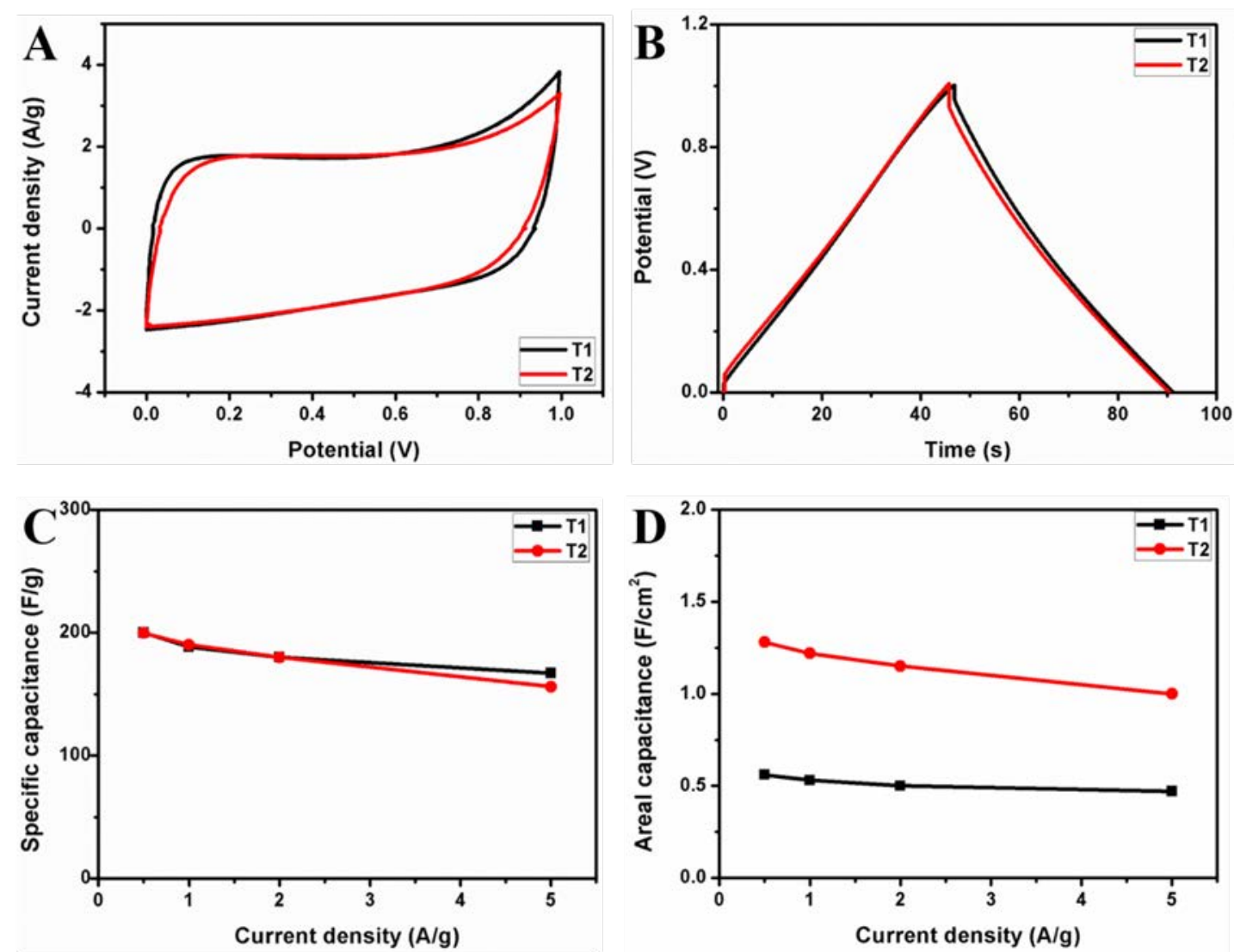

Figure 5. Electrochemical performance of 3D SMG electrodes with different mass loadings (T1 for $2.8 \mathrm{mg} / \mathrm{cm}^{2}$ and T2 for $6.4 \mathrm{mg} / \mathrm{cm}^{2}$ ): (A) CV curves at scan rate of $20 \mathrm{mV} / \mathrm{s}$, (B) galvanostatic charge/discharge profiles at current density of $2 \mathrm{~A} / \mathrm{g},(\mathrm{C})$ current densities vs. specific capacitances, and (D) areal capacitances at different current densities. 
3D surface-microporous graphene, in which meso/macro channels are formed by graphene walls and rich micropores are incorporated in the surface layer of the graphene walls, is an ideal electrode material for a supercapacitor. It achieves an ultrahigh areal capacitance of $1.28 \mathrm{~F} / \mathrm{cm}^{2}$ and a high gravimetric capacitance of $200 \mathrm{~F} / \mathrm{g}$, three times larger than that of commercially used activated carbon.

Keywords: cabbage-coral-like graphene, surface micropores, electric double-layer capacitors, ultrahigh areal capacitance

Liang Chang, Dario J. Stacchiola and Yun Hang Hu*

An Ideal Electrode Material, 3D Surface-microporous Graphene for Supercapacitors with Ultrahigh Areal Capacitance

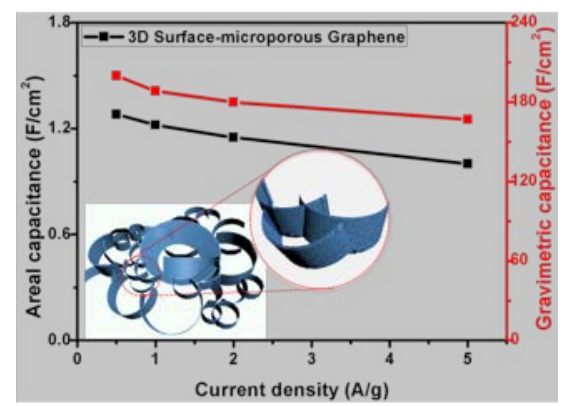




\section{Supporting Information}

An Ideal Electrode Material, 3D Surface-microporous Graphene for Supercapacitors with Ultrahigh Areal Capacitance

Liang Chang, Dario J. Stacchiola, Yun Hang Hu*

Content

1. Experimental section

2. Characterization of SMG

3. Characterization of activated carbon

4. Calculation of average micropore deepness of SMG 


\section{Experimental section}

\subsection{Synthesis of 3D surface-microporous graphene}

Metal Na cubes were loaded into a ceramic tube reactor and 50 psi $\mathrm{CO}_{2}$ was introduced into the reactor at room temperature. Then, the reactor was heated to $520^{\circ} \mathrm{C}$ and the temperature was maintained for $12 \mathrm{~h}$. Finally, the obtained products were treated with $37 \mathrm{wt} \% \mathrm{HCl}$, followed by deionized water washing for several times until $\mathrm{PH}=7$ and drying at $80^{\circ} \mathrm{C}$ oven for overnight. The obtained carbon materials are donated as surface-microporous graphene (SMG).

\subsection{Characterization of 3D surface-microporous graphene (SMG)}

The structure of SMG was evaluated by Field emission scanning electron microscope (FESEM, Hitachi-4700) with energy dispersive spectroscopy (EDS) and transmission electron microscope (TEM, JEOL JEM2010F). X-ray diffraction (XRD) was used to determine the crystal structures of solid products with a Scintag XDS-2000 powder diffract meter (Cu Ka $(\lambda=1.5406 \AA)$ ). Pore size distribution and surface area of SMG were measured by $\mathrm{N}_{2}$ adsorption/desorption at liquid nitrogen temperature (77 K) with ASAP 2000 instrument. The composition and functional groups of SMG were characterized by Fourier transform infrared (FTIR) spectra (a Perkin Elmer Spectrum One with $\mathrm{KBr}$ pellets method) and X-ray photoelectron spectroscopy (XPS, a Kratos Ultra AXIS DLD XPS with a monochromated Al source).

\subsection{Fabrication of electrodes and performance test of electric double-layer capacitors}

The SMG electrode was fabricated as follows: SMG, carbon black (conductive additive), and poly(tetrafluoroethylene) (binder) were fully mixed with 80:10:10 weight ratio in isopropyl alcohol to form a homogeneous paste. Then the paste was rolled to a rectangular strip and pressed on $1 \mathrm{x} 1 \mathrm{~cm}^{2}$ nickel foam (as current collector). The obtained electrode was dried in $80^{\circ} \mathrm{C}$ oven for 24 h to get rid of organic solvent of binder and isopropyl alcohol. For comparison purpose, the same procedure was exploited to fabricate the activated carbon electrode with activated carbon (AC) (Aldrich Chemical Company, In). 
The electric double-layer performance was tested with symmetrical two-electrode configuration, in which the SMG electrodes (or AC electrodes) were used as both cathode and anode, a glassy microfiber filter (Whatman, GF/F) as separator located between the electrodes, and $2 \mathrm{M} \mathrm{KOH}$ aqueous solution as electrolyte. The assembled supercapacitor cells were subjected to cyclic voltammetry (CV) tests and galvanostatic charge/discharge measurements in the potential range of $0 \sim 1 \quad \mathrm{~V}$ with electrochemical workstation (Princeton Potentiostat/Galvanostat Model 273A). The scan rates of CV curves were $30 \sim 300 \mathrm{mV} / \mathrm{s}$, and the current density of charge/discharge profiles were $0.5 \sim 10 \mathrm{~A} / \mathrm{g}$. Electrochemical impedance spectroscopy (EIS) was conducted at frequency of $0.01 \sim 10^{5} \mathrm{~Hz}$ with three-electrode configuration, in which working electrode, counter electrode, and reference electrode are SMG electrode (or AC electrode), Pt electrode, and saturated $\mathrm{Hg} / \mathrm{Hg}_{2} \mathrm{Cl}_{2}$ electrode, respectively.

\section{Characterization of SMG}

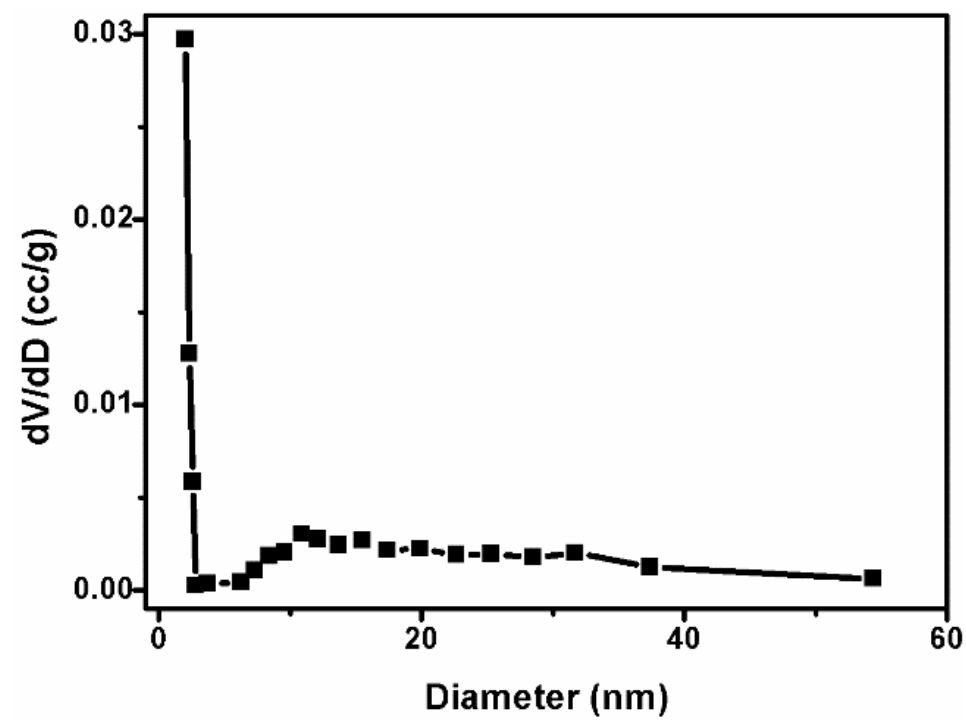

Figure S1. Pore size distribution of SMG. 

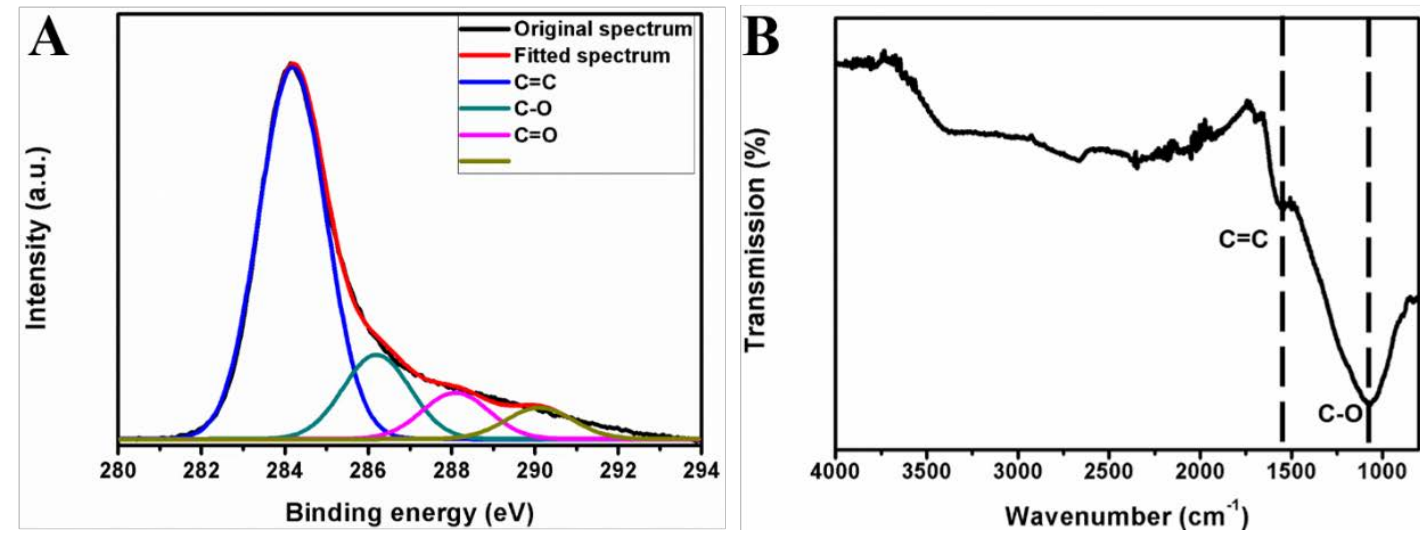

Figure S2. Characterization of 3D SMG: (A) XPS spectra and (B) FTIR spectrum.

\section{Characterization of activated carbon}
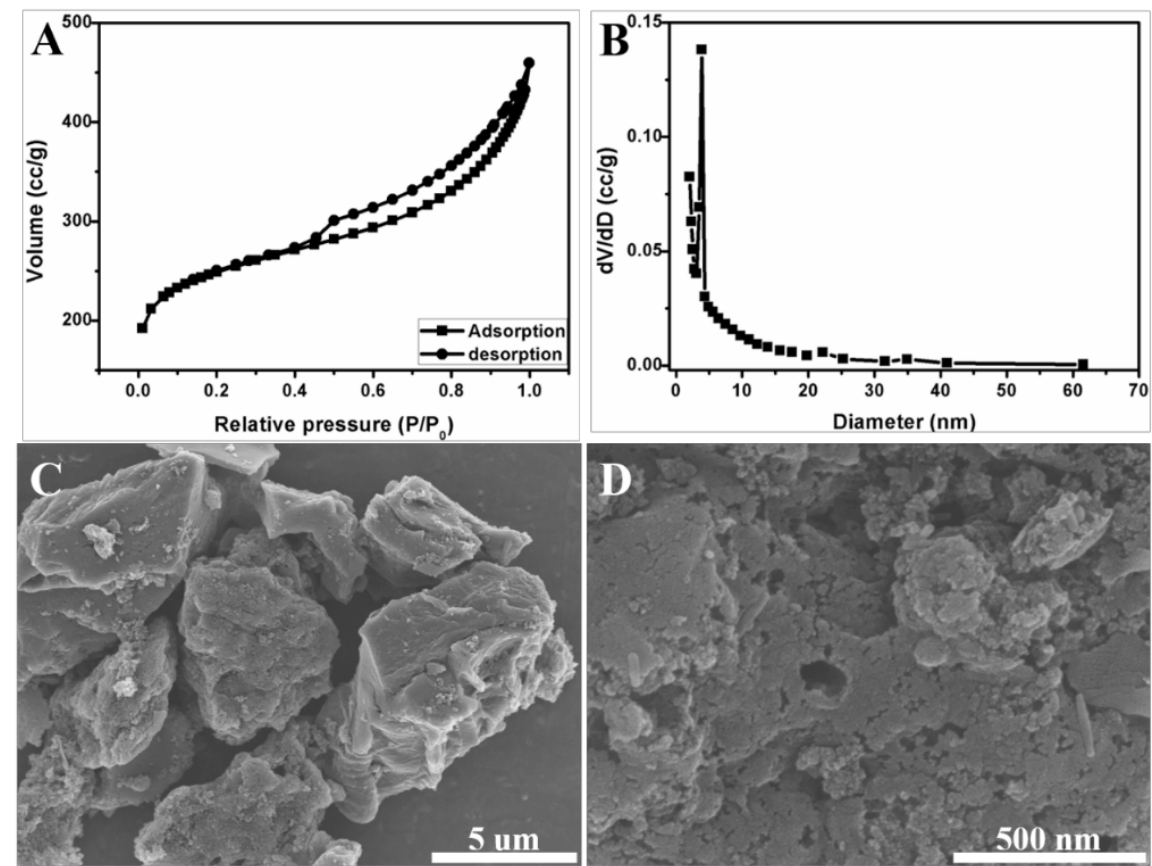

Figure S3. Characterization of activated carbon, (A) $\mathrm{N}_{2}$ adsorption/desorption curves, (B) pore size distribution, (C) and (D) SEM images.

Activated carbon (AC, 100 mesh) was purchased from Aldrich Chemical Company, Inc. Its surface area and pore size distribution were measured with $\mathrm{N}_{2}$ adsorption at $77 \mathrm{~K}$. In Figure S3A, the $\mathrm{N}_{2}$ adsorption/desorption curves present type II isotherm with a type $\mathrm{H} 3$ hysteresis loop. The surface area calculated from BET model is $853 \mathrm{~m}^{2} / \mathrm{g}$ in which $500 \mathrm{~m}^{2} / \mathrm{g}$ is 
from micropore areas, indicating $\mathrm{AC}$ is a microporous material. The pore size distribution shown in Figure S3B further demonstrated that the pore sizes of the most pores are smaller than $5 \mathrm{~nm}$. Microstructure of AC was shown by FESEM images (Figure S3C and S3D).

\section{Calculation of average micropore-deepness of SMG}

The theoretic surface area $\left(S_{\text {theory }}\right)$ of one obtain graphene sheet (consisting of 6 graphene layers) without any pores can be calculated as

$$
S_{\text {theory }}=\frac{2630}{6}=438\left(\mathrm{~m}^{2} / \mathrm{g}\right)
$$

When micropores are considered as cylinder pores, the wall surface area (excluding bottom areas) of the micropores in graphene sheets can be calculated as

$$
S_{\text {wall }}=S_{\text {total }}-S_{\text {theory }}=890-438=452\left(\mathrm{~m}^{2} / \mathrm{g}\right)
$$

The external surface area $\left(S_{e x}\right)$ of graphene sheets, which includes the surface area of meso/macro channels, can be calculated as

$$
S_{\text {ex }}=S_{\text {total }}-S_{\text {micropores }}=890-789=101\left(\mathrm{~m}^{2} / \mathrm{g}\right)
$$

The average diameter (D) of micropores is $1.6 \mathrm{~nm}$. Furthermore, we can also express $S_{e x}$ as

$$
S_{\text {ex }}=S_{\text {theory }}-n \pi\left(\frac{D}{2}\right)^{2}
$$

where $\mathrm{n}$ is the number of micropores in a gram graphene. Therefore

$$
n=\frac{S_{\text {theory }}-S_{\text {ex }}}{\pi\left(\frac{D}{2}\right)^{2}}=\frac{438-101}{3.14\left(\frac{1.6 \times 10^{-9}}{2}\right)^{2}}=1.68 \times 10^{20}(1 / g)
$$

The introduction of $S_{\text {wall }}, n, h$, and $D$ values into Equation 1 gives the average deepness $(h)$ of micropores as

$$
h=0.54(n m)
$$

